\title{
On the Shock Front Thickness in Water and other Molecular Liquids
}

\author{
Siegfried Hess \\ Institut für Theoretische Physik, Technische Universität Berlin, PN 7-1, \\ Hardenbergstr. 36, D-10623 Berlin
}

Z. Naturforsch. 52 a, 213-219 (1997); received October 29, 1996

\begin{abstract}
Theoretical explanations are presented for the deviation of the shock front thickness from linear hydrodynamics, as observed by W. Eisenmenger (1964) in water and several molecular liquids for large driving pressure differences. Two mechanisms are proposed, which are based on generalizations of the Maxwell relaxation equation for the friction pressure tensor. One is due to the spatial inhomogeneity and linked with piezo-electric or piezo-tetradic effects. The other is caused by nonlinearities which account for shear thickening.
\end{abstract}

\section{Introduction}

Within the range of applicability of (linear) hydrodynamics, the thickness of a shock front (shock width) in a fluid is closely related to the damping of sound waves, which, in turn, is determined by the transport coefficients of thermo-hydrodynamics, viz.: the viscosity, the bulk viscosity and the thermal conductivity [1]. The first measurements of the shock width in liquids which were reliable enough to test the hydrodynamic theory, showed satisfactory agreement for small driving pressure differences but significant devations for larger pressure differences [2]. The shock width is larger than the corresponding hydrodynamic value. The transport coefficients seem to increase with increasing deviations from equilibrium. In this article, two mechanisms are discussed which can account for the observed deviations from linear hydrodynamis. These are effects associated with the spatial inhomogeneity of the friction pressure tensor and with a nonlinear generalization of Maxwell's relaxation model, recently invented to describe shear thickening [4]. Though these ideas have been presented previously at conferences [3], it has been over thirty years since the publication of the article by W. Eisenmenger [2], which still contains the best experimental data for water and some other molecular liquids. Nonequilibrium molecular dynamics (NEMD) computer simulations, performed for a simple Lennard-Jones model liquid, yielded similar deviations from hydro-

Reprint requests to Prof. S. Hess, e-mail: S.Hess@physik.tu-berlin.de. dynamics [5], yet at high temperatures and for driving pressure differences orders of magnitude larger than those used in the experiments.

This article proceeds as follows. In Sect. 2, the pressure profile in the shock front and the meaning of the shock width $L$ are stated. The expression for $L$ derived from linear hydrodynamics is reviewed and a function $H$ is introduced which characterizes the relative deviation of $L$ from it. Experimental results for water and methanol as measured by Eisenmenger [2] are presented graphically. Section 3 is devoted to the discussion of two conjectures for the explanation of the observed deviations from hydrodynamics. Both employ generalizations of the Maxwell relaxation equation for the friction pressure tensor. The first one (Sect. 3.1) is the addition of a term proportional to a second spatial derivative of the pressure tensor. This leads to a relative deviation $H$ of the shock width from its hydrodynamic value which is proportional to the square of the driving pressure difference. The plot of the data for water and methanol confirm such a behavior within the experimental uncertainties. A new characteristic length parameter $\ell$, which turns out to be a few hundred to one thausand times larger than the size of a molecule, can be infered from this analysis. Some speculations on the origin of the additional term in the Maxwell relaxation equation and the meaning of the length $\ell$ are presented. A link with piezo-electric or piezo-tetradic effects is indicated. The second mechanism (Sect. 3.2) is caused by terms nonlinear in the friction pressure tensor. In lowest order in the nonlinearity, again it is found that $H$ is proportional to the square of the driving pressure

0932-0784 / 97 / 0300-0213 \$ 06.00 @ - Verlag der Zeitschrift für Naturforschung, D-72072 Tübingen 


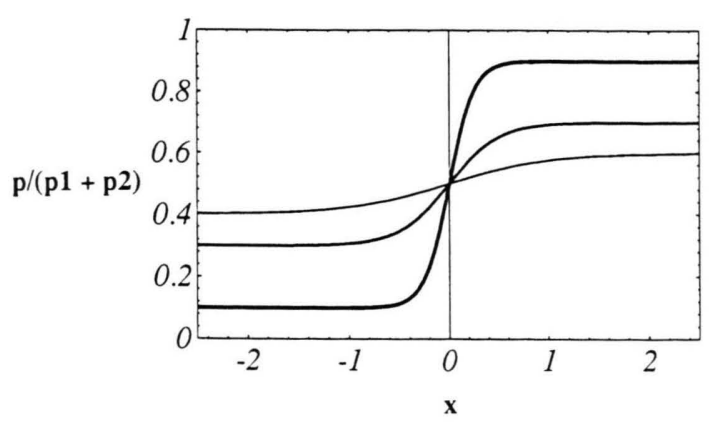

Fig. 1. Pressure profile in the vicinity of the shock front.

difference. Now, however, the proportionality coefficient has a different meaning. Results from the full nonlinear theory [4] are also compared with the experimental data for water. Good agreement is found. Some concluding remarks are added.

\section{Basics and Motivation}

\subsection{Pressure Profile}

The pressure $p=p(x)$ in the vicinity of a plane shock wave, propagating in the $-x$-direction, is, in a co-moving coordinate system where $x=0$ corresponds to the center of the shock front, given by [1]:

$$
p=\frac{1}{2}\left(p_{2}+p_{1}\right)+\frac{1}{2}\left(p_{2}-p_{1}\right) \tanh (2 x / L) .
$$

Here $\delta p=p_{2}-p_{1}$ is the pressure difference driving the shock wave, $p_{1}$ and $p_{2}$ are the asymptotic values of the pressure before and behind the shock front. The shock width is denoted by $L$. The pressure $p(x)$, in units of the average pressure $\left(p_{1}+p_{2}\right) / 2$, is depicted schematically in Fig. 1 as function of $x$ in units of a convenient reference length. The thickness $L$ decreases with increasing driving pressure $\delta p$. More specifically, the shock width can be written as

$$
L=H L_{\text {ref }} p_{\text {ref }}(\delta p)^{-1},
$$

where the product of the reference values for the length and pressure, $L_{\text {ref }}$ and $p_{\text {ref }}$, is determined by linear hydrodynamics. The quantity $H=H\left(\delta p / p_{\text {ref }}\right)$, with $H=1$ in the hydrodynamic limit, characterizes the deviation from linear hydrodynamics.

In the experiments, the shock width is inferred from the pressure rise time $t_{\text {rise }}=L / c_{\mathrm{S}}$ in the shock front, and $c_{\mathrm{S}}$ is the speed of the shock wave, which is practically equal to the adiabatic sound velocity [2].

\subsection{Hydrodynamics}

Linear thermo-hydrodynamics [1] yields (2) with $H=1$, and

$$
L_{\text {ref }} p_{\text {ref }}=16 a V^{2}\left(\frac{\partial^{2} V}{\partial p^{2}}\right)_{\mathrm{s}}^{-1}
$$

with the abbreviation

$$
a=\frac{V}{2 c_{\mathrm{S}}^{3}}\left[\left(\frac{4}{3} \eta+\eta_{\mathrm{V}}\right)+\lambda\left(\frac{1}{c_{\mathrm{v}}}-\frac{1}{c_{\mathrm{p}}}\right)\right] .
$$

Here $V=\rho^{-1}$ is the specific volume and $c_{\mathrm{S}}$ the sound velocity, The shear viscosity, bulk viscosity and heat conductivity are denoted by $\eta, \eta_{\mathrm{V}}$, and $\lambda$, respectively, $c_{\mathrm{v}}$ and $c_{\mathrm{p}}$ are the specific heat at constant volume and constant pressure.

The decrease of the amplitide $\operatorname{Ampl}(x)=\operatorname{Ampl}(0)$ $\exp (-\alpha x)$ of a sound wave with frequency $\omega=2 \pi \nu$, propagating in $x$-direction, is characterized by the inverse length parameter $\alpha$, which is related to the quantity $a$ occuring in (3) and (4) by $\alpha=\omega^{2} a$. By order of magnitude, one has $a \approx 10^{-15} \mathrm{~s}^{2} \mathrm{~m}^{-1}$ for water and the other liquids studied in [2].

\subsection{The Experiment}

In $1964 \mathrm{~W}$. Eisenmenger published data on the shock width for liquid water, acetone, methanol, ethanol, a few other organic substances, as well as for some mixtures, in the article [2] with the title Experimentelle Bestimmung der Stossfrontdicke aus dem akustischen Frequenzspektrum elektromagnetisch erzeugter Stosswellen in Flüssigkeiten bei einem Stossdruckbereich von $10 \mathrm{~atm}$ bis $100 \mathrm{~atm}$ (Experimental determination of the shock front thickness from the acoustic frequency spectrum of electromagnetically generated shock waves in liquids in the shock pressure range of $10 \mathrm{~atm}$ to $100 \mathrm{~atm} ; 1 \mathrm{~atm}$ $\approx 10^{5} \mathrm{~Pa}$ ). In Figs. 2 and 3 , his data are used to plot the shock front thickness $L$, in units of the conveniently chosen reference length $L_{\text {ref }}=1 \mu \mathrm{m}$, versus the pressure difference $\delta p$ for water and methanol. The quantity $\delta p$ is expressed in units of a reference pressure $p_{\text {ref }}$, which is determined by the hydrodynamic value as it follows from (3) and (4) with the 


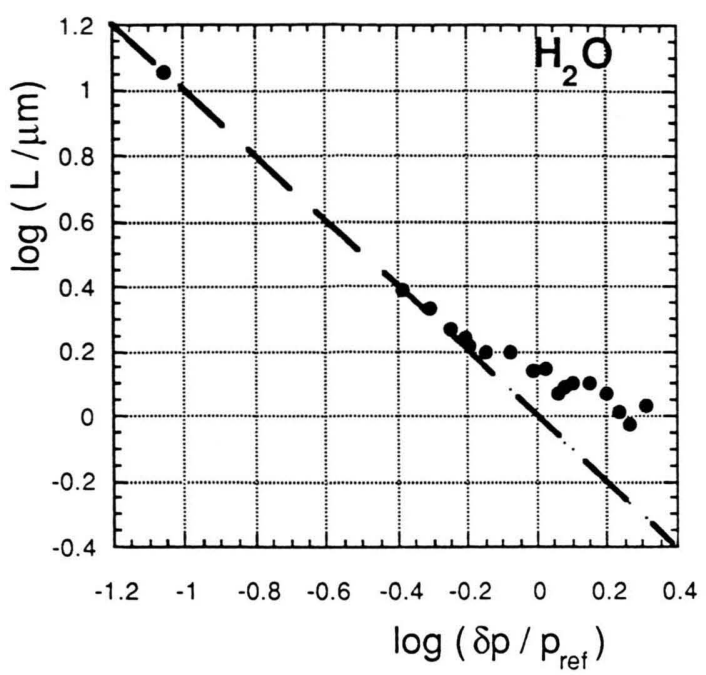

Fig. 2. The shock front thickness $L$ in water as function of the presssure difference $\delta p, L_{\text {ref }}=1 \mu \mathrm{m}, p_{\text {ref }} \approx 70 \mathrm{~atm}$. Data from Eisenmenger [2].

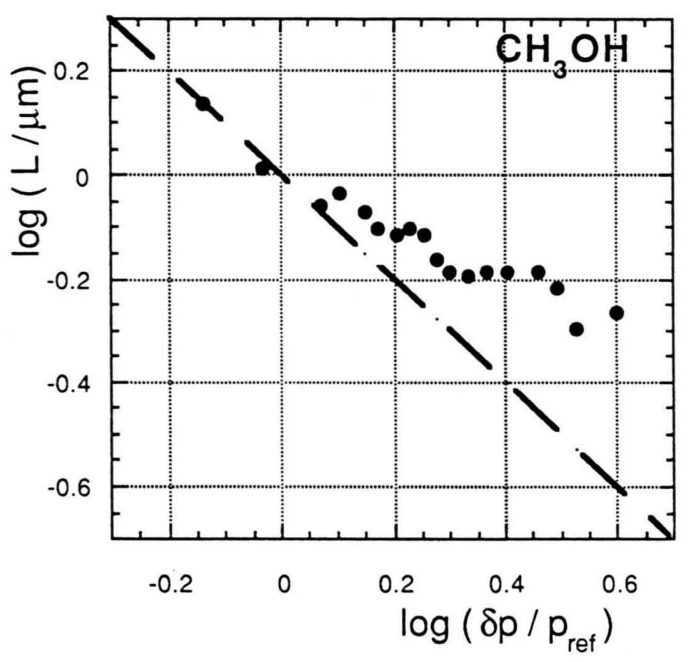

Fig. 3. The shock front thickness $L$ in methanol as function of the presssure difference $\delta p, L_{\text {ref }}=1 \mu \mathrm{m}, p_{\text {ref }} \approx 17 \mathrm{~atm}$. Data from Eisenmenger [2].

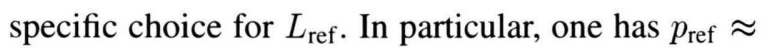
$70 \mathrm{~atm}$ and $p_{\text {ref }} \approx 17 \mathrm{~atm}$ for water and methanol, respectively. The straight (dashed) lines correspond to the hydrodynamic result. No adjustable parameters occur in the comparison with the experimental data. The hydrodynamic limit is approached for small values of $\delta p$, Significant deviations, however, are ob-

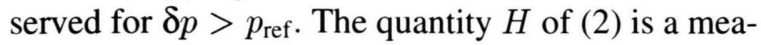
sure of this deviation. Notice that the shock width is two to four orders of magnitude larger than a molecular length. Thus a continuum description of the shock problem is appropriate.

\section{Deviations from Hydrodynamics}

The density and temperature dependence of the transport coefficients and of the other thermo-physical properties within the shock front account for the deviations of the shock width from linear hydrodynamics, as observed in the strong shock waves of the NEMD simulations [5] for simple fluids. This explanation does not work for the relatively weaker shocks employed in the experiments with the molecular liquids [2] to be analyzed here.

Additional sources for deviations from hydrodynamics are modifications of the constitutive relations between the thermodynamic forces and fluxes, govering the transport processes. Here emphasis is put on the friction pressure tensor and the shear viscosity. Similar modifications for the bulk viscosity and the heat conductivity, which may well be of importance, are not treated explicitly.

Consequences of two generalizations of the Maxwell relaxation equation for the friction pressure tensor, associated with spatial inhomogeneities and with terms nonlinear in the friction pressure, are presented.

\subsection{Spatial Inhomogeneity}

The friction pressure tensor $\boldsymbol{\pi}=\overleftrightarrow{\mathbf{p}}$, i. e. the symmetric traceless part of the pressure tensor $\mathbf{p}$ (negative stress tensor) is assumed to obey the generalized Maxwell relaxation equation

$$
\tau_{\mathrm{M}} \frac{\partial}{\partial t} \pi+\ell^{2} \Delta \pi+\pi=-2 \eta \overleftrightarrow{\nabla v}
$$

The symbol $\longleftrightarrow$ indicates the symmetric traceless part of a tensor. The Maxwell relaxation time $\tau_{\mathrm{M}}$ is related to the (newtonian) shear viscosity $\eta$ by $\eta=G \tau_{\mathrm{M}}$, where $G$ is the high frequency shear modulus. In (5), $\Delta$ is the Laplacian and $\ell$ stands for a characteristic length which is considered as a model parameter. Arguments for the derivation of this additional term are given later. When spatial inhomogeneities of the friction pressure tensor over the length $\ell$ are small, the term $\ell^{2} \Delta \pi$ can be disregarded. Then (5) reduces to the time honoured standard Maxwell relaxation equation. 
When the rise time $t_{\text {rise }}=L / c_{\mathrm{S}}$ of the pressure in the shock front is large compared to the Maxwell relaxation time $\tau_{\mathrm{M}}$, it suffices to analyze the stationary solution

$$
\pi=-2 \eta \overleftrightarrow{\nabla v}-\ell^{2} \Delta \pi
$$

Considering the term involving the length $\ell$ as a small perturbation, one obtains, in lowest order in $\ell^{2}$,

$$
\pi=\approx-2 \eta\left(1-\ell^{2} \Delta\right) \overleftrightarrow{\nabla v}
$$

The continuity equation, for a stationary state, $\partial\left(\rho v_{x}\right) / \partial x=0$, implies

$$
\begin{aligned}
\partial v_{x} / \partial x & =-\rho^{-1} v_{x}(\partial \rho / \partial p)_{\mathrm{S}} \partial p / \partial x \\
& =\rho^{-1} c_{\mathrm{S}}^{-1} \partial p / \partial x .
\end{aligned}
$$

Notice that $c_{\mathrm{S}}^{2}=(\partial p / \partial \rho)_{\mathrm{S}}$. Again in lowest order in $\ell^{2}$, one has

$$
\ell^{2} \Delta \partial v_{x} / \partial x \approx-(\ell / L)^{2} \partial v_{x} / \partial x .
$$

Thus the viscosity $\eta$ occuring in the expression (4) which, in turn, determines the shock front thickness $L$, can be replaced by the effective viscosity

$$
\eta_{\mathrm{eff}}=\eta\left(1+(\ell / L)^{2}\right) .
$$

Assuming, for simplicity, that all transport coefficients are modified in the same manner, the quantity $H$, specifying the deviation of $L$ from its hydrodynamic value, is given by

$$
H:=\frac{L \delta p}{L_{\text {ref }} p_{\text {ref }}}=1+\left(\frac{\ell}{L_{\text {ref }}}\right)^{2}\left(\frac{\delta p}{p_{\text {ref }}}\right)^{2}
$$

In Figs. 4 and 5, $H=L \delta p$, in units of $L_{\text {ref }} p_{\text {ref }}$, is plotted versus $\left(\delta p / p_{\text {ref }}\right)^{2}$ for water and methanol. The points represent the same data as shown in Figs. 2 and 3. Within the experimental uncertainty, $H$ increases in direct proportion to $(\delta p)^{2}$ in accord with (11). From the slope of the dashed straight lines one inferes $\left(\frac{\ell}{L_{\text {ref }}}\right)^{2} \approx 0.3, \approx 0.08$ for $\mathrm{H}_{2} \mathrm{O}$ and $\mathrm{CH}_{3} \mathrm{OH}$, respectively. Thus the characteristic length parameter $\ell$, for water and methanol, is approximately equal to $0.5 \mu \mathrm{m}$ and $0.3 \mu \mathrm{m}$. Of course, with all the approximations made here, these numbers should just be regarded as an estimate of the order of magnitude

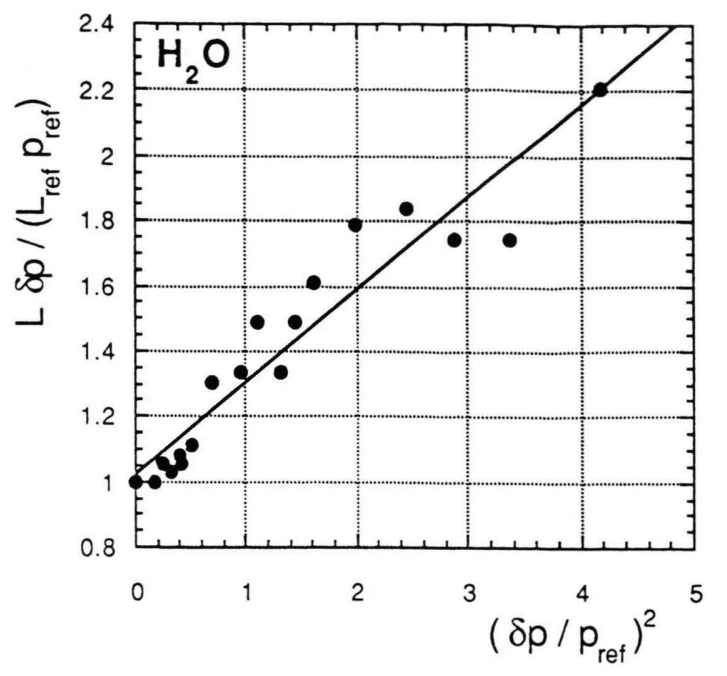

Fig. 4. Shock front thickness times pressure difference $L \delta p$ in water as function of the square of the pressure difference $(\delta p)^{2}, L_{\text {ref }}=1 \mu \mathrm{m}, p_{\text {ref }} \approx 70 \mathrm{~atm}$. Data from Eisenmenger [2].

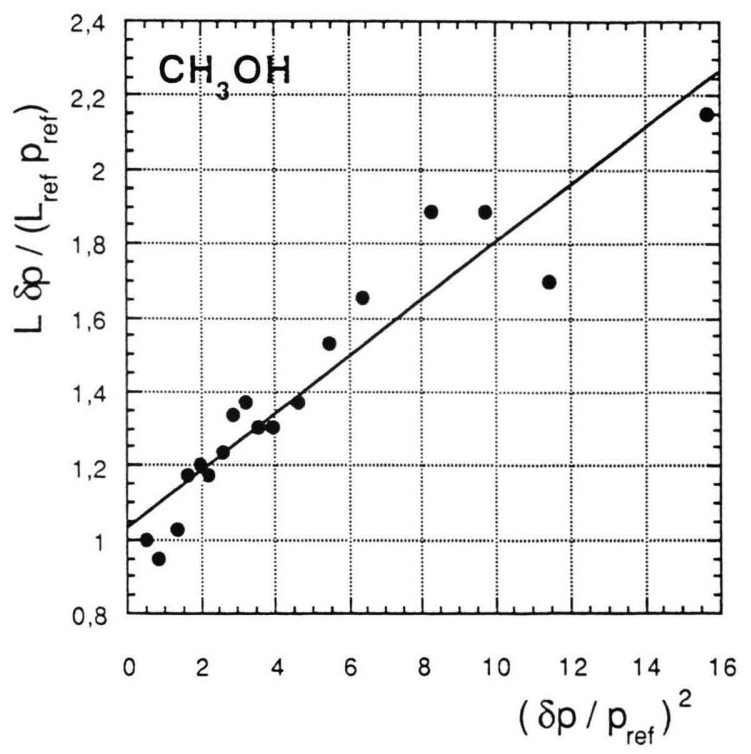

Fig. 5. Shock front thickness times pressure difference $L \delta p$ in methanol as function of the square of the pressure difference $(\delta p)^{2}, L_{\text {ref }}=1 \mu \mathrm{m}, p_{\text {ref }} \approx 17 \mathrm{~atm}$. Data from Eisenmenger [2].

of $\ell$. Nevertheless, one is now faced with the question "where does a characteristic length come from which is several hundred to one thousand times larger than the size of a molecule?". 


\subsubsection{Origin of the length parameter $\ell$}

Various causes can lead to an additional term in the Maxwell relaxation equation which involve the Laplacian. These are

1) diffusive processes,

2) energetic effects in analogy to Frank elasticity of liquid crystals,

3) piezo-electric and piezo-tetradic couplings.

Cause 1), referred to as the "Burnett" contribution in connection with gases [6], can be ruled out because the characteristic length involved there is of the order of a mean free path in gases, or of the order of a molecular size in liquids [7] and, even more important, because of an opposite sign in front of $\ell^{2}$ in (5). Burnett-like terms would imply a decrease of the shock width with increasing driving pressure difference rather than the observed increase. The same remark with regard to the sign applies to cause 2) unless one makes the rather unlikely assumption that a spatially inhomogeneous state of the friction pressure tensor is energetically more favourable than a homogeneous one. Cause 3), favoured here, deserves some additional explanation. The relaxation equation for the friction pressure tensor $\pi$ is written in the form

$$
\tau_{\mathrm{M}} \frac{\partial}{\partial t} \pi+\stackrel{\leftrightarrow}{\Phi}=-2 \eta \overleftrightarrow{\nabla v}
$$

where $\overleftrightarrow{\Phi}$ is the derivative of a Landau type potential function $\Phi$ with respect to $\pi$. The simple choice $\Phi=(1 / 2) \pi: \pi$, e. g. yields $\stackrel{\leftrightarrow}{\Phi}=\pi$ corresponding to the standard Maxwell model. The above mentioned piezo-electric and piezo-tetradic couplings mean the use of the Landau potential

$$
\begin{aligned}
\Phi=\frac{1}{2} \boldsymbol{\pi}: \boldsymbol{\pi} & +\frac{1}{2} \alpha_{1} \boldsymbol{d} \cdot \boldsymbol{d}+\frac{1}{2} \alpha_{3} \mathbf{t}: \mathbf{t} \\
& +\zeta_{1} \boldsymbol{\pi}:(\overleftrightarrow{\nabla \boldsymbol{d}})+\zeta_{3} \boldsymbol{\pi}:(\boldsymbol{\nabla} \cdot \mathbf{t}) .
\end{aligned}
$$

Here the vector $\boldsymbol{d}$ and the third rank tensor $\mathbf{t}$ are the dipolar and the tetradic order parameters associated with the dipole moment and the tetrahedral coordination of the molecules. The quantities $\alpha_{1}>0, \alpha_{3}>0$, and $\zeta_{1,3}$ are phenomenological coefficients. The latter ones, which may have either sign, characterize the coupling between the friction pressure tensor, the dipolar and the tetradic order parameters. The potential (13) implies

$$
\overleftrightarrow{\Phi}=\pi+\zeta_{1} \overleftrightarrow{\nabla d}+\zeta_{3} \nabla \cdot \mathbf{t}
$$

In analogy to (12) with (14), the dipolar order parameter obeys the relaxation equation

$$
\tau_{1} \frac{\partial}{\partial t} \boldsymbol{d}+\alpha_{1} \boldsymbol{d}-\zeta_{1} \boldsymbol{\nabla} \cdot \boldsymbol{\pi}=0
$$

where the relaxation time $\tau_{1}$ is an additional phenomenological coefficient. In a stationary situation, one obtains

$$
\boldsymbol{d}=\frac{\zeta_{1}}{\alpha_{1}} \nabla \cdot \pi
$$

which underlies the piezo-electric effect. A similar relation, now involving the ratio $\zeta_{3} / \alpha_{3}$, is found for the tetradic order parameter. Insertion of these expressions for $\boldsymbol{d}$ and $\mathbf{t}$ into (14) leads to terms involving second spatial derivatives of the friction pressure tensor which, for the geometry under consideration, are equivalent to the Laplacian used in (5). Then one has, apart from factors of the order of 1 ,

$$
\ell^{2}=\frac{\zeta_{1}^{2}}{\alpha_{1}}+\frac{\zeta_{3}^{2}}{\alpha_{3}} .
$$

Hence the piezo-electric or piezo-tetradic coupling yields the correct sign irrespective of the sign of $\zeta_{1}$ and $\zeta_{3}$. No estimate of the order of magnitude of the coefficients occuring on the r.h.s. of (17) can be given. The coefficients $\alpha_{1,3}$ in the denominator of (17) could become quite small, and consequently $\ell^{2}$ rather large, due to collective effects as encountered in pretransformational phenomena, e.g. in liquid crystals [8], [9]. Consequences of the piezo couplings for the sound absorption should be analyzed.

A test of the importance of the piezo effects expected in the polar liquids could be provided by a comparison with data for liquids where such effects do not exist due to the symmetry of the molecules. Indeed, the only substance of that kind measured by Eisenmenger [2], viz. liquid $\mathrm{CCl}_{4}$, does not show any deviation from the preditions of linear hydrodynamics. Since the range of driving pressure differences was rather limited in that case, no definitive conclusions can be drawn, however. 


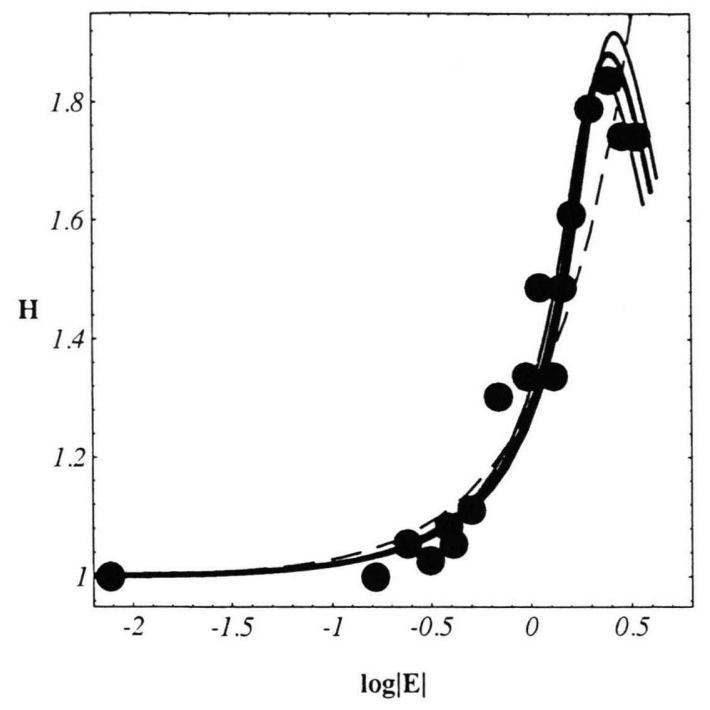

Fig. 6. The relative deviation from hydrodynamics $H \sim$ $L \delta p$ as function of the logarithm of the dimensionless extension rate $E \sim(\delta p)^{2}$. Comparison with the experimental data for water (Eisenmenger [2]) with curves of the full nonlinear theory (full curves) and the simple approximation (dashed).

\subsection{Nonlinearity}

In general, the relaxation equation for the friction pressure tensor $\pi$ will also contain terms nonlinear in $\pi$. Disregarding effects associated with spatial inhomogeneities, the generalized Maxwell relaxation equation

$$
\begin{aligned}
\tau_{\mathrm{M}} \frac{\partial}{\partial t} \pi+\ldots & +\pi-\sqrt{6} B \overleftrightarrow{\pi \cdot \pi} \\
& +C \pi(3 \pi: \pi)=-2 \eta \overleftrightarrow{\nabla v}
\end{aligned}
$$

has been used to study the nonliear flow behavior for simple shear, planar biaxial and uniaxial flows [4]. The ellipses ... stand for terms involving products of components of the velocity gradient tensor with $\pi$ which, however, are disregarded here. Depending on the values of the model parameters $B$ and $C$, both shear thinning and shear thickening were found. It should be mentioned that a slightly different scaling of the variables, e.g. $\pi$ expressed in units of the high frequency shear modulus, was used in [4]. Here, the nonlinear relaxation equation is applied for the appropriate uniaxial extensional or compressional flow. As before, the extension rate is $\epsilon=\partial v_{x} / \partial x \approx-\rho^{-1} c_{\mathrm{S}}^{-1}(\delta p / L) \sim-(\delta p)^{2}$. In lowest order in the nonlinearity $(C=0)$ one finds, for a stationary situation,

$$
H:=\frac{L \delta p}{L_{\text {ref }} p_{\text {ref }}}=1+\frac{B p_{\text {ref }} \eta}{\rho c_{\mathrm{S}} L_{\text {ref }}}\left(\frac{\delta p}{p_{\text {ref }}}\right)^{2} .
$$

Again, the deviation of $H$ from its value 1 based on linear hydrodynamics is directly proportional to $(\delta p)^{2}$. Thus the slope of the straight lines in Figs. 4 and 5 can be used to determine the coefficient $B$. For water one finds $B \approx 6(\mathrm{~atm})^{-1}$.

In Fig. 6 the experimental data of [2] for water, in particular $H \sim L \delta p$ plotted versus the logarithm of the dimensionless extension rate $E \sim(\delta p)^{2}$, are compared with curves of the full nonlinear theory (full curves) where $C / B^{2}=0.52,0.53,0.54$. The agreement seems to be rather good. The data point of Fig. 4 at the largest value of $\delta p$, however, is not included in Figure 6. The dashed curve follows from the simple approximation $C=0$, which yields (19).

\section{Concluding Remarks}

Two mechanisms have been presented which can account for the experimentally observed deviation of the shock width from its (linear) hydrodynamic value. For specific cases, the relevant new phenomenological coefficients have been inferrred from the data upon the assumption that one process dominates. To predict the relative importance of these two contributions, however, independent experimental data or estimates from model calculations are needed for the coefficients determining the magnitude of the effects discussed here. Non-equilibrium molecular dynamics (NEMD) computer simulations of shock waves in molecular liquids could also be helpful to clarify this issue

\section{Acknowledgement}

I thank W. Eisenmenger for having, a long time ago, drawn my attention to his shock wave experiments and for reminding me, over many years, at the Spring meetings of the Deutsche Physikalische Gesellschaft (DPG), that the observed deviations of the shock front thickness from hydrodynamics requires a theoretical explanation. 
[1] L. D. Landau und E. M. Lifschitz, Lehrbuch der Theoretischen Physik, Bd. VI, Hydrodynamik, Akademie-Verlag, Berlin 1966, p. 394 ff.; Fluid Mechanics, Pergamon Press, London 1959.

[2] W. Eisenmenger, Acustica 14, 187 (1964).

[3] 150 Jahre DPG, 59. Physikertagung Berlin 1995, Verhandl. DPG(IV) 30, 1115 (1995); EPS, 15th General Conference of the Condensed Mater Division, Baveno-Stresa 1996, Europhysics Conference Abstracts 20 A, 265 (1996).

[4] O. Hess and S. Hess, Physica A 207, 517 (1994); in: StatPhys 19, ed. Hao Bailin, World Scientific, Singapore 1996 , p. 369.

[5] W. G. Hoover, Phys. Rev. Lett. 42, 1531 (1979); B. L. Holian, W. G. Hoover, B. Moran, and G. K. Straub, Phys. Rev. A 22, 2798 (1980); B. L. Holian, in: Microscopic Simulations of Complex Hydrodynamic
Phenomena, eds. M. Mareschal and B.L. Holian, Plenum, New York 1992, p. 75.

[6] S. Chapman and T. G. Cowling, Mathematical Theory of Nonuniform Gases, University Press, Cambridge 1964; L. Waldmann, in: Handbuch der Physik, Vol. XII, ed. S. Flügge, Springer-Verlag, Berlin 1959.

[7] G. Schmidt, W. E. Köhler, and S. Hess, Z. Naturforsch. 36 a, 545 (1981).

[8] P. G. de Gennes, The Physics of Liquid Crystals, Clarendon Press, Oxford 1974; H. Kelker and R. Hatz, Handbook of Liquid Crystals, Verlag Chemie, Weinheim 1980; G. Vertogen and W. deJeu, Thermotropic Liquid Crystals, Fundamentals, No. 45 in Chemical Physics, Springer-Verlag, Berlin 1988.

[9] S. Hess, Z. Naturforsch. 30a, 728, 1224 (1975); 31a, 1034, 1507 (1976). 